\title{
Autologous ilium graft combination with Y-shaped titanium plate fixation for chest wall reconstruction after resection of primary sternal tumors - a clinical study from three institutions
}

\author{
Shuonan Xu' ${ }^{1,2 \#}$, Yawei Dou ${ }^{1 \#}$, Guolong Zhao, ${ }^{1,3 \#}$, Jianfei Zhu ${ }^{1}$, Wei Tian ${ }^{1}$, Wei Sun ${ }^{4}$, Zongzhi Liu ${ }^{5}$, \\ Lanjun Zhang ${ }^{6,7}$, Hongtao Wang ${ }^{1}$
}

${ }^{1}$ Department of Thoracic Surgery, Shaanxi Provincial People's Hospital, Xi'an 710068, China; ${ }^{2}$ Department of Cardiothoracic Surgery, The First Affiliated Hospital of Xi'an Medical University, Xi'an 710077, China; ${ }^{3}$ Department of Cardiovascular Surgery, The Northwest Women and Children Hospital, Xi'an 710061, China; ${ }^{4}$ Department of Thoracic Surgery, The Affiliated Tumor Hospital of Xinjiang Medical University, Urumqi 830054, China; ${ }^{5}$ Department of Orthopedics, Shaanxi Provincial People's Hospital, Xi'an 710068, China; ${ }^{6}$ State Key Laboratory of Oncology in South China, Collaborative Innovation Center for Cancer Medicine, Guangzhou 510060, China; ${ }^{7}$ Department of Thoracic Surgery, Sun Yat-sen University Cancer Center, Guangzhou 510060, China

Contributions: (I) Conception and design: L Zhang, H Wang; (II) Administrative support: H Wang; (III) Provision of study materials or patients: J Zhu, W Sun, L Zhang; (IV) Collection and assembly of data: S Xu, Y Dou, G Zhao; (V) Data analysis and interpretation: S Xu, Y Dou, G Zhao; (VI) Manuscript writing: All authors; (VII) Final approval of manuscript: All authors.

\#These authors contributed equally to this work.

Correspondence to: Lanjun Zhang. State Key Laboratory of Oncology in South China, Collaborative Innovation Center for Cancer Medicine, Guangzhou 510060, China; Department of Thoracic Surgery, Sun Yat-sen University Cancer Center, Guangzhou 510060, China. Email: zhanglj@sysucc.org.cn; Hongtao Wang. Department of Thoracic Surgery, Shaanxi Provincial People’s Hospital, Xi'an 710068, China. Email: whtsyy139@sina.com.

Background: The technique for anterior chest wall reconstruction after resection of primary sternal tumors (PST) still continue to evolve.

Methods: A total of 12 PST patients from three hospitals who underwent en-bloc resection were included in our study. After finishing sternum resection, autologous iliac bone combined with Y-shaped titanium plate were applied to rebuild the anterior chest wall. Postoperative outcomes were analyzed.

Results: There were 10 different types of tumors located in manubrium (6 cases), sternum body (4 cases) and Louis's angle ( 2 cases) in our research. For these patients, the median resected tumor size and the area of defect after sternal resection were $279.0 \mathrm{~cm}^{3}$ and $215.0 \mathrm{~cm}^{2}$, respectively. The mean operative time was $299.2 \pm 65.2 \mathrm{~min}$ and intra-operative blood loss was $431.2 \pm 213.0 \mathrm{~mL}$. Mean duration of drainage was $9.9 \pm 2.6$ days. In their perioperative period, significant circulatory and respiratory complications occurred in 8 patients. Postoperative chest X-ray and tridimensional CT images showed autogenous reconstruction of the sternum and titanium in good position. No side effects were observed 6-12 months post reconstructive surgery, but one patient suffered from anchor loss and prosthesis migration. Y-shaped titanium plates from two patients were separately removed at 24 and 26 months when the reconstructive sternum integrated with skeleton anterior chest wall well.

Conclusions: Our study demonstrates the safety and feasibility of this new technique for anterior chest wall reconstruction after sternectomies.

Keywords: Autologous ilium graft; titanium plate; primary sternum tumors; resection; reconstruction

Submitted Aug 21, 2019. Accepted for publication Nov 29, 2019.

doi: $10.21037 /$ tcr.2019.12.36

View this article at: http://dx.doi.org/10.21037/tcr.2019.12.36 


\section{Introduction}

Primary sternal tumors (PST) are uncommon in clinical practice, which only account for $15 \%$ of total chest wall tumors (1,2). En-bloc resection $(3,4)$, is still the major therapeutic strategy for PST. However, after the sternal resection, reconstruction of the chest wall brings us more challenge for filling defects, stabilizing chest wall and minimizing respiratory impairment, which is vital to postoperative quality of life.

Traditionally, the reconstruction materials of skeletal framework deprived from the following aspects: (I) autologous materials: autologous iliac had been employed for reconstruction to sustain chest wall stability $(5,6)$, the infection and rejection are minimal and the effects of the reconstruction are physiological due to the material derived from the patients' tissues $(7,8)$, however, autologous materials were limited for the wide chest wall defect. (II) Synthetic soft materials: including Marlex meshes (9), Prolene meshes (10), polytetrafluoroethylene (PTFE) patches and so on. The advantages of the synthetic soft materials were availability and ease of use. The disadvantage was patients underwent chest wall reconstruction occurred paradoxical respiration more frequently. (III) Synthetic hard materials: such as titanium mesh, titanium plate and plexiglass, it was necessary to use this kind of materials when the defect was large $(1,11)$. The materials were widely used in clinical practice but every material has its own limitation.

The PST can be divided into primary sternum manubrium tumors (PSMT) and primary sternum body tumors (PSBT) according to the tumor location. For patients with PST, after the sternal resection, reconstruction of the chest wall is important to ensure good acromioclavicular joint function, adequate rigidity, minimal complications. Thus we applied a new surgical technique for sternum reconstruction using autologous ilium graft combination with Y-shaped titanium plate fixation.

\section{Methods}

\section{Case collection}

From January 2006 to December 2017, 32 patients diagnosed as PST from three medical institutions were collected in our research. All patients underwent systematic preoperative evaluations, including enhanced computed tomography (CT) scan (Figure 1), echocardiography, electrocardiogram, pulmonary function test and bone scintigraphic imaging, to be confirmed that whether their cardiopulmonary function were satisfied with surgical indication and no removal metastasis. The criterion of selection for this surgical approach is that pre-operative CT images and whole-body bone scanning revealed primary tumor located in sternum (including manubrium, sternum body and Louis's angle) without metastasis occurred in our cases. Finally, 16 patients were enrolled for further analysis and 12 of them received synchronous sternum resection and chest wall reconstruction, their clinical-pathological features were listed in Table 1.
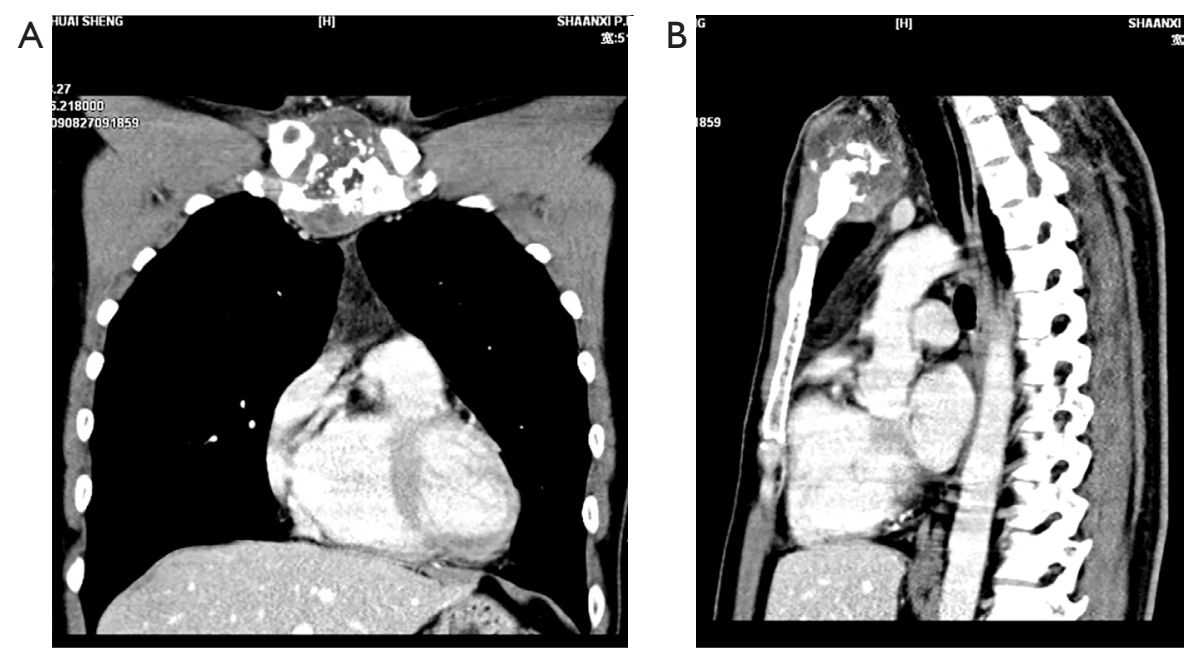

Figure 1 Tumor located at sternum by computed tomography scan. (A) Coronal scan; (B) sagittal scan. 
Table 1 Clinicopathological features of enrolled patients

\begin{tabular}{lccccc}
\hline Patient No. & Age (years) & Gender & Chief complain & Tumor location & Maxim diameter in CT (cm) \\
\hline 1 & 52 & Male & Chest pain & Manubrium & Manubrium \\
2 & 59 & Female & Chest pain & Manubrium & 9.0 \\
3 & 56 & Male & Chest and right shoulder pain & Sternum body & 9.5 \\
4 & 71 & Female & Chest pain & Sternum body & 12.0 \\
5 & 55 & Male & Chest pain & Louis's angle & 4.2 \\
6 & 22 & Female & Chest pain & Manubrium & 7.5 \\
7 & 37 & Male & Physical examination & Louis's angle & 11.0 \\
8 & 58 & Male & Chest pain & Manubrium & 7.5 \\
9 & 68 & Male & Physical examination & Sternum body & 16.0 \\
10 & 52 & Female & Chest pain, chest tightness & Sternum body & \\
11 & 34 & Female & Chest pain & Manubrium & 4.0 \\
\hline
\end{tabular}

\section{Surgical approach}

\section{Sternum resection}

$E n-b l o c$ resection (12), including wide resection of the affected part of the sternum plus closely adjacent tissues, had clear margins of at least $2.0 \mathrm{~cm}$ were advocated to minimize the risk of local recurrence. Sternectomy was defined (13) as (I) total, when no sternal remnant was present after resection, (II) subtotal, when $2 / 3$ or more of the sternum was removed, and (III) partial, when less than $2 / 3$ of the sternum was removed. Based on the range of lesion, the unilateral or bilateral ribs, clavicular heads, even partial pericardium were removed for establishing clear margins.

\section{Chest wall reconstruction \\ Model Y-shaped titanium plate}

The angle of the upper arms of Y-shaped titanium plate (Suzhou Dual Sheep Medical Technology Company, China) was regulated to match with the bilateral clavicle, then fold sternal angle on the third arm of Y-shaped titanium (sternal angle measured by preoperative for shaping). Each patient had received Y-shaped titanium plate with different specifications. The original titanium plates were produced from factory with three different size. According to the area and maximum diameter of defect, we choose the reasonable Y-shaped titanium plate for our patients. By trimming surplus from distal plate and adjusting the angle between the arms and plane, the modified titanium plate was deployed, and steal nails were fixed with them. To ensure the stability and well-implantable of fixation, the size of titanium plate was designed as covering more than one third of width of sternal body, proximal clavicle and two-thirds of width of manubrium, also a certain number of circular holes were reserved for steel nails penetration.

\section{Autogenous iliac bone graft and implantation}

According to the size and contour of the chest wall defect, patients' unilateral or bilateral anterior iliac spine were removed from autogenous pelvis and tailored to padding the defect area. Next, holes were drilled $0.1 \mathrm{~cm}$ from the end of the ribs and grafted ilium, the polydioxanone sutures (Johnson \& Johnson Medical Devices Company, USA) were employed to fix the grafted ilium and the host rib stumps in end-to-end fashion. Moreover, the Y-shaped titanium wings fixed with bilateral clavicles, and the other side fixed with the autogenous graft or remaining sternum. Driving steel nails into the skeleton frame to be combined with the Y-shaped titanium plate. At last, bridged the gap between the sternum and clavicle using the iliac bone wedge to make the connection more closely (Figure 2). After the sternum or chest drainage tube placed, the muscle and skin were closed routinely.

After finishing the surgery, in order to stabilize the chest wall, patients" chest was compressed and fixed by " 8 " resin bandage and chest strap (bilateral shoulder joint must be at adduction) for more than one month, also the postoperative mobilization of patients' upper limbs was strictly refrained at least two weeks. 


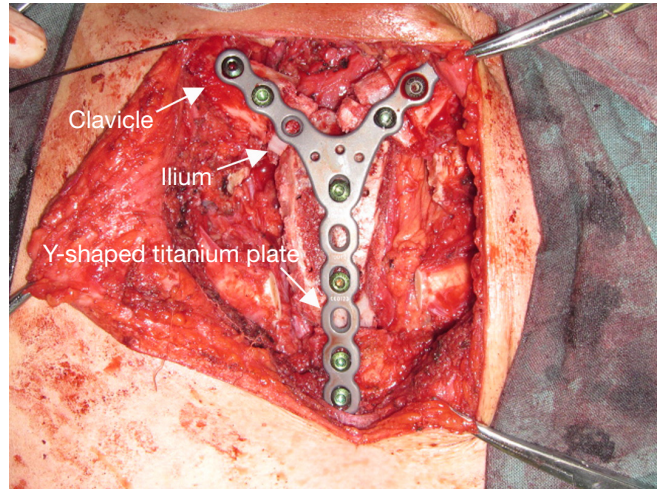

Figure 2 The new technique of sternum resection and reconstruction using Y-shaped titanium plate and autogenous ilium graft.

\section{Results}

We reviewed 12 cases ( 7 males and 5 females) with a median age of 53.5 years for this study. The collected data showed that 9 patients (75\%) admitted to chest pain and 10 types of benign or malignant tumors confirmed by histopathologic diagnosis were located in manubrium (6 cases), sternum body ( 4 cases) and Louis's angle ( 2 cases). The median resected tumor size was $279.0 \mathrm{~cm}^{3}$ and the area of defect after sternal resection was $215.0 \mathrm{~cm}^{2}$ (the shape of the tumor is irregular, so we measured the maximum length of tumor on sagittal axis, coronal axis and vertical axis, respectively). Furthermore, the mean operative time was $299.2 \pm 65.2 \mathrm{~min}$, intra-operative blood loss was $431.2 \pm 213.0 \mathrm{~mL}$ and mean duration of drainage was $9.9 \pm 2.6$ days. In their perioperative period, postoperative complications occurred in 8 patients, which included pleural effusion (66.7\%), pulmonary infection $(16.7 \%)$, atelectasis $(8.3)$; prosthesis loosing $(8.3 \%)$, atrial fibrillation $(16.7 \%)$ and dermatic necrosis $(8.3 \%)$. The surgical information and postoperative complications were demonstrated in Table 2. Postoperative chest X-ray and tridimensional CT images showed autogenous reconstruction of the sternum and titanium in good position (Figure 3). The criteria of the prosthesis removal included: (I) 3D reconstruction of chest $\mathrm{CT}$ showed that graft integrated with skeleton anterior chest wall well at least one year after operation; (II) no local relapse or metastasis happened before the surgery of removal arrangement. (III) Patients strongly desired to remove the prosthesis by surgery due to pain or discomfort. In our research, 2 patients suited the principles, so their prosthesis were removed. Y-shaped titanium plate were respectively removed at 24 months (patient No. 2) and 26 months (patient No. 11) when the reconstructive sternum integrated with skeleton part of anterior chest wall well (Figure 4).

Unfortunately, a male patient (patient No. 3) underwent prosthesis migration as a result of nails loosing confirmed by CT scan on the sixth day after surgery, two days later we refixed the prosthesis by flexible steel wires. Following pneumonia, pleural effusion with symptomatic treatment for one month after second operation, the patient recovered and discharged from hospital.

\section{Discussion}

For treating PST, many scholars devoted themselves to explore a more simplified and feasible surgical approach of synchronously sternal resection and chest wall reconstruction. In Suzuki et al.'s retrospection (14), both traditional materials (such as meshes and patches) and newer techniques (such as custom-made titanium implant and scapular autograft) were fully analyzed. In earlier, McCormack et al. (15) applied materials consisted of Marlex mesh and methyl methacrylate to reconstruct chest wall after malignant tumors were resected. There is a general consensus among surgeons that 'sandwich' technique is a standard method to repair defected chest wall. Through sequential repair pleura, bone skeleton and muscles, rigidity of the chest wall was ensured and sternoclavicular joint and end of ribs were anchored. Kużdżał et al. (16) used two-tier of heavy polypropylene mesh with methyl methacrylate bone cement between them to finish major part of 'sandwich reconstruction'. But for this classic reconstructive technique, badly tight and unelastic features might lead to forming dead space, effusion and refractory infection. So including titanium plate, autologous rib, mesh or any other reconstructive materials, no one was recommended to be the optimal material.

In recent years, 3D-print technique is frequently applied for skeletal structure reconstruction, through equal-ratio stimulating actual size of defected sternum or ribs, chest wall was restored accurately and suitably $(17,18)$. Wang et al. (19) applied 3D-print titanium ribs to complex reconstruct chest wall defect after extensive chest wall resection for a 60-year-old male with T3-stage non-small cell lung cancer. Also some Chinese scholars (20) reported 18 cases receiving $3 \mathrm{D}$-print chemical composed materials for chest wall reconstruction, Polyether-ether-ketone was used to customize prosthesis for implanting into chest wall. As a result, 10 costectomies and 8 cases of sternal resection carried out synchronous chest wall reconstruction.

Although 3D-print technique presented more accurate, self-adaptable and rigid features in restoring defected chest 


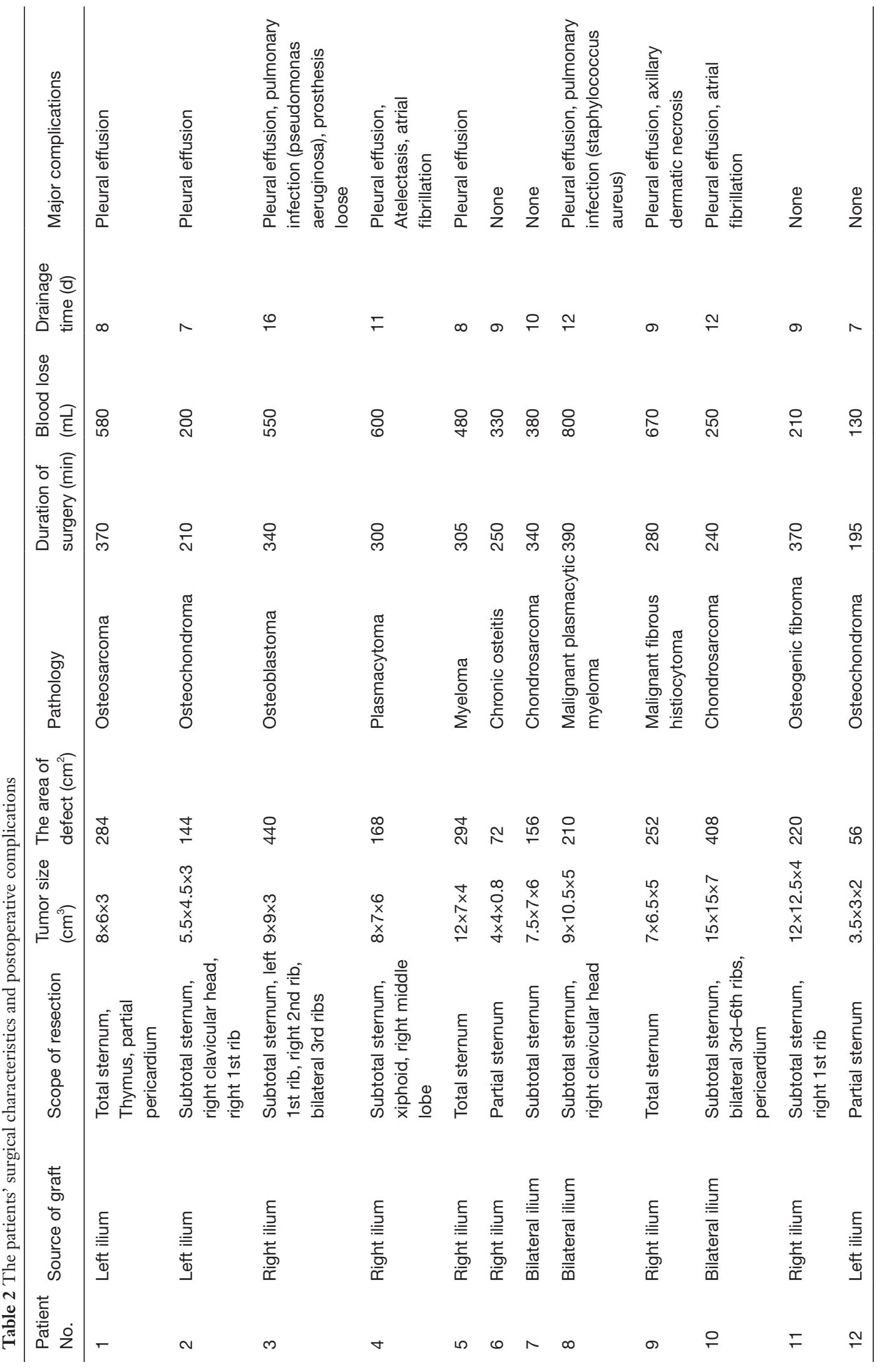



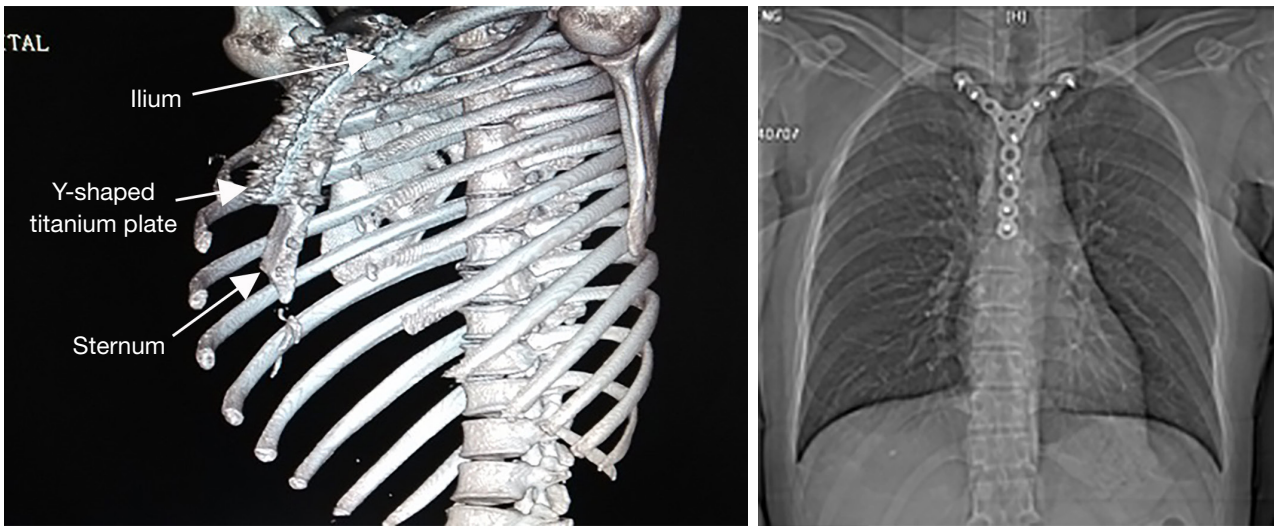

Figure 3 A tridimensional computed tomography scan shows the sternum reconstruction at 6 weeks.

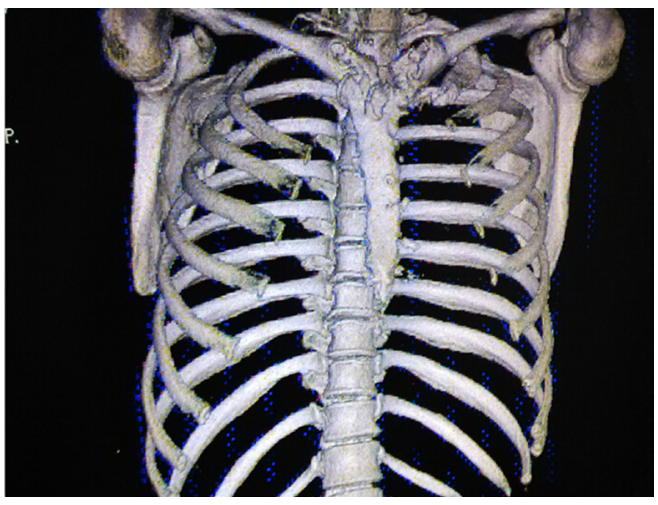

Figure 4 A tridimensional computed tomography scan shows Y-shaped titanium plate were removed at 26 months.

wall, drawbacks of this technique are obvious. Complex procedure of 3D-print model production means high cost and long preoperative preparation, meantime a high degree of integration lower the activity of small joints, which may reduce respiratory mobility. Autologous materials have clear advantages such as easy acquirement and low expense that autograft could be popularized and practical. In the area of plastic and reconstructive surgery, autologous rib cartilage, illum or other bone tissue were used for repair trachea (21), nose (22) and auricle (23). Zhang et al. (24) used autologous rib grafts to reconstruct the defected chest wall after manubrium tumors resection, and extubation finished within 24 hours and no perioperative mortality occurred during treatment. However, for this technique, harvesting ribs had a risk of chest wall malacia, which might lead to abnormal respiration. In our study, we utmostly minimized the application of biomaterials and used autologous ilium grafts to avoid potential immune rejection as well as reduce the medical cost. With the assistance of Y-shaped titanium plate anchoring, the stability and rigid of restored chest wall was guaranteed. Importantly, the autograft became a real member of chest wall after removing titanium plate so that chest-based complications were reduced and the quality of life was improved.

The drawbacks of our reconstruction technique should be mentioned. Prosthesis loosing happened in a case receiving subtotal sternal, clavicular head and multiple ribs resection, which showed autologous ilium grafts were difficult in matching area of huge thoracic defection, and sampling areas were limited. But this study for autologous ilium graft combined with Y-shaped titanium plate fixation for chest wall reconstruction for treating PST is meaningful and reliable. In the future, large-scale cohort study should be designed to further surveillance of safety for this reconstruction technique and long-term outcomes.

\section{Acknowledgments}

Funding: This study was supported by Wu Jieping Medical Foundation (320.6750.17527).

\section{Footnote}

Conflicts of Interest: All authors have completed the ICMJE uniform disclosure form (available at http://dx.doi. org/10.21037/tcr.2019.12.36). The authors have no conflicts of interest to declare.

Ethical Statement: The authors are accountable for all aspects of the work in ensuring that questions related to the accuracy or integrity of any part of the work are appropriately investigated and resolved. The study was conducted in accordance with the Declaration of Helsinki (as revised in 
2013). This study was approved by the Ethics Committee of Shaanxi Provincial People's Hospital (No. B2019-028-13) and written informed consent was obtained from all patients.

Open Access Statement: This is an Open Access article distributed in accordance with the Creative Commons Attribution-NonCommercial-NoDerivs 4.0 International License (CC BY-NC-ND 4.0), which permits the noncommercial replication and distribution of the article with the strict proviso that no changes or edits are made and the original work is properly cited (including links to both the formal publication through the relevant DOI and the license). See: https://creativecommons.org/licenses/by-nc-nd/4.0/.

\section{References}

1. Wu X, Chen M, Yu F. Application of titanium plate and Teflon patch in chest wall reconstruction after sternal. tumor resection. Zhongguo Xiu Fu Chong Jian Wai Ke Za Zhi 2011;25:1224-6.

2. Martini N, Huvos AG, Burt ME, et al. Predictors of survival in malignant tumors of the sternum. J Thorac Cardiovasc Surg 1996;111:96-105; discussion 105-6.

3. Meyer $M$, Neef H, Zerkowski HR. Alloplastic sternal replacement in malignant sternal tumors. Case report and review of the literature. J Cardiovasc Surg (Torino) 1998;39:853-7.

4. Incarbone $M$, Nava $M$, Lequaglie C, et al. Sternal resection for primary or secondary tumors. J Thorac Cardiovasc Surg 1997;114:93-9.

5. Cara JA, Lacleriga AF, Canadell J. Iliac allograft used for sternal reconstruction after resection of a chondrosarcoma. Int Orthop 1993;17:297-9.

6. Ren P, Zhang J, Zhang X. Resection of primary sternal osteosarcoma and reconstruction with homologous iliac bone: case report. J Formos Med Assoc 2010;109:309-14.

7. Tunçözgür B, Elbeyli L, Gungor A, et al. Chest wall reconstruction with autologas rib grafts in dogs and report of a clinic case. Eur J Cardiothorac Surg 1999;16:292-5.

8. Chai Y, Zhang G, Shen G. Autogenous rib grafts for reconstruction of sternal defects after partial resection: a new surgical technique. Plast Reconstr Surg 2008;121:353e-355e.

9. Zhang Y, Li JZ, Hao YJ, et al. Sternal tumor resection and reconstruction with titanium mesh: a preliminary study. Orthop Surg 2015;7:155-60.

10. Baqain EB, Lataifeh IM, Khriesat WM, et al. Primary repair of a large incomplete sternal cleft in an asymptomatic infant with Prolene mesh. J Pediatr Surg 2008;43:e39-41.

11. Demondion P, Mercier O, Kolb F, et al. Sternal replacement with a custom-made titanium plate after resection of a solitary breast cancer metastasis. Interact
Cardiovasc Thorac Surg 2014;18:145-7.

12. Chapelier A. Resection and reconstruction for primary sternal tumors. Thorac Surg Clin 2010;20:529-34.

13. Girotti P, Leo F, Bravi F, et al. The "rib-like" technique for surgical treatment of sternal tumors: lessons learned from 101 consecutive cases. Ann Thorac Surg 2011;92:1208-15; discussion 1215-6.

14. Suzuki K, Jones DR. Sternal Resections: Conventional and Novel Materials for Reconstruction. Curr Surg Rep 2015;3:17.

15. McCormack P, Bains MS, Beattie EJ Jr, et al. New trends in skeletal reconstruction after resection of chest wall tumors. Ann Thorac Surg 1981;31:45-52.

16. Kużdżał J, Warmus J, Grochowski Z, et al. Reconstruction of the sternal manubrium. J Thorac Cardiovasc Surg 2014;147:1986-8.

17. Lipińska J, Kutwin L, Wawrzycki M, et al. Chest reconstruction using a custom-designed polyethylene 3D implant after resection of the sternal manubrium. Onco Targets Ther 2017;10:4099-103.

18. Bertin F, Deluche E, Tricard J, et al. First case of sternum replacement with a bioceramic prosthesis after radioinduced sarcoma. Curr Oncol 2018;25:e351-3.

19. Wang L, Cao T, Li X, et al. Three-dimensional printing titanium ribs for complex reconstruction after extensive posterolateral chest wall resection in lung cancer. J Thorac Cardiovasc Surg 2016;152:e5-7.

20. Wang L, Huang L, Li X, et al. Three-dimensional printing PEEK implant: a novel choice for the reconstruction of chest wall defect. Ann Thorac Surg 2019;107:921-8.

21. Xu S, Zhu J, Zhao G, et al. Tracheal suspension with autogenous rib cartilage in a patient with severe tracheomalacia. J Cardiothorac Surg 2019;14:21.

22. Agostini T, Lazzeri D, Pascone C, et al. Onlay bone autografts in nasal augmentation: ilium versus calvaria. Plast Reconstr Surg 2010;126:1787-8; author reply 1788-9.

23. Xu Z, Zhang R, Zhang Q, et al. New Strategies for Remnant Ear Treatment in Lobule-Type Microtia Reconstruction. Plast Reconstr Surg 2018;142:471-9.

24. Zhang G, Liang C, Shen G, et al. Autogenous rib grafts for reconstruction of the manubrium after resection: technical refinements and outcomes. J Thorac Cardiovasc Surg 2014;148:2667-72.

Cite this article as: Xu S, Dou Y, Zhao G, Zhu J, Tian W, Sun W, Liu Z, Zhang L, Wang H. Autologous ilium graft combination with Y-shaped titanium plate fixation for chest wall reconstruction after resection of primary sternal tumorsa clinical study from three institutions. Transl Cancer Res 2020;9(2):930-936. doi: 10.21037/tcr.2019.12.36 